\title{
Efeitos da música clássica aplicada em crianças hospitalizadas
}

\author{
Effects of classic music applied to hospitalized children
}

\section{Efectos de la música clásica aplicada a los niños hospitalizados}

Ana Célia Pereira da Silva ${ }^{1 *}$, Marcelo Lima de Oliveira ${ }^{2}$, Leonardo Cesar Carvalho², Ciderleia Castro de Lima'.

\section{RESUMO}

Objetivo: Verificar os efeitos da música clássica em crianças hospitalizadas em Unidade de Terapia Intensiva e Enfermaria de Pediatria. Métodos: É um estudo quase experimental, abordagem quantitativa. Participaram quatro crianças menores de 36 meses, expostas a 40 minutos diários por cinco dias ao repertório Mozart baby, respeitando a altura de som de 45 decibéis (dB). Utilizou um formulário estruturado para registros de frequência cardíaca, frequência respiratória, saturação, temperatura, escala de dor. Os critérios de inclusão foram a exposição a procedimentos invasivos nas últimas seis horas, frequência inspiratória de oxigênio $\left(\mathrm{FiO}_{2}\right)$ menor ou igual a $21 \%$ e ventilação não assistida. Aplicado no músculo bíceps braquial um eletrodo e analisado a tensão muscular no primeiro e quinto dia. $O$ registro feito pelo eletromiografo Trigno 8 Channel Wireless. A exposição ao estresse foi avaliado pela saliva em fita teste no primeiro e quinto dia, no aparelho Coroco Meter (Nipro Corporation - Japão). Resultados: Houve redução nos valores hemodinâmicos com p-valor menor que 0,01 , a dor com $p$-valor menor que 0,001. A eletromiografia verificou redução dos impulsos elétricos influenciando na taxa do disparo para as fibras musculares com relaxamento muscular. Conclusão: A música estimula diferentes áreas cerebrais e pode ser utilizada para auxiliar segmentos terapêuticos.

Palavras-chave: Enfermagem, Humanização da assistência, Hospitalização.

\begin{abstract}
Objective: To verify the effects of classical music in children hospitalized in the Intensive Care Unit and Pediatric Ward. Methods: It is a quasi-experimental study, a quantitative approach. Four children under 36 months participated, exposed to 40 minutes daily for five days to the Mozart baby repertoire, respecting the loudness of 45 decibels $(\mathrm{dB})$. It used a structured form to record heart rate, respiratory rate, saturation, temperature, pain scale. Inclusion criteria were exposure to invasive procedures in the last six hours, oxygen inspiratory frequency ( $\mathrm{FiO} 2$ ) less than or equal to $21 \%$ and unattended ventilation. An electrode was applied to the biceps brachii muscle and muscle tension was analyzed on the first and fifth days. The registration made by the electromyography Trigno 8 Channel Wireless. Exposure to stress was assessed by test tape saliva on the first and fifth days, on the Coroco Meter (Nipro Corporation - Japan). Results: There was a reduction in hemodynamic values with $p$-value less than 0.01 , pain with $p$-value less than 0.001 . Electromyography verified a reduction in electrical impulses, influencing the rate of firing for muscle fibers with muscle relaxation. Conclusion: Music stimulates different brain areas and can be used to assist therapeutic segments.
\end{abstract}

Keywords: Nursing, Humanization of assistance, Hospitalization.

1 Universidade José do Rosário Vellano (UNIFENAS). Alfenas - MG. *E-mail: aninhajl2008@gmail.com

2 Universidade Federal de Alfenas (UNIFAL). Alfenas - MG.

SUBMETIDO EM: 2/2020

ACEITO EM: $3 / 2020$

PUBLICADO EM: 5/2020

REAS/EJCH | Vol.Sup.n.48 | e3215 | DOI: https://doi.org/10.25248/reas.e3215.2020 Página 1 de 9 


\section{RESUMEN}

Objetivo: Verificar los efectos de la música clásica en niños hospitalizados en la Unidad de Cuidados Intensivos y Sala de Pediatría. Métodos: Un estudio cuasi-experimental, un enfoque cuantitativo. Participaron cuatro niños menores de 36 meses, expuestos a 40 minutos diarios durante cinco días al repertorio para bebés de Mozart, respetando el volumen de 45 decibelios (dB). Utilizó una forma estructurada para registrar la frecuencia cardíaca, la frecuencia respiratoria, la saturación, la temperatura y la escala del dolor. Los criterios de inclusión fueron exposición a procedimientos invasivos en las últimas seis horas, frecuencia inspiratoria de oxígeno (FiO2) menor o igual al $21 \%$ y ventilación desatendida. Aplicó un electrodo al músculo bíceps braquial y analizó la tensión muscular en el primer y quinto día. El registro realizado por la electromiografía Trigno 8 Channel Wireless. La exposición al estrés se evaluó mediante cinta de prueba de saliva en el primer y quinto día, en el medidor Coroco (Nipro Corporation - Japón). Resultados: Hubo una reducción en los valores hemodinámicos con un valor $p$ menor que 0.01 , dolor con un valor $p$ menor que 0.001 . La electromiografía verificó una reducción en los impulsos eléctricos, influyendo en la velocidad de disparo de las fibras musculares con la relajación muscular. Conclusión: La música estimula diferentes áreas del cerebro y puede usarse para ayudar a segmentos terapéuticos.

Palabras clave: Enfermeria, Humanización de la atención, Hospitalización.

\section{INTRODUÇÃO}

O ser humano, quando se depara com uma situação de enfermidade, seja dele próprio ou de um ente querido, começa a olhar a realidade de maneira diferente, sendo que as emoções, os sentimentos e as incertezas até então desconhecidos, começam a ser experimentados. Principalmente em se tratando de crianças, de acordo com a gravidade do quadro clínico e havendo necessidade de internação em unidade de terapia intensiva (UTI), é comum que surja sensações de estranheza, impotência, stress e temor na criança e nos pais (MOLINA RCM, et al., 2014).

Ao ser encaminhado para UTI o paciente e os seus familiares percebem a gravidade de seu quadro clínico, o que possibilita desencadear aspectos emocionais que podem culminar em crise de sentimentos e até mesmo estresse (BATISTA MA, 2004). Em ambiente hospitalar é visto que as crianças não têm muitas opções lúdicas e são expostas diariamente a situações estressantes o que pode influenciar em seu desenvolvimento infantil (CASTRO NETO A, 2000).

Desta forma, ao incentivar e facilitar os pacientes a expressarem seus sentimentos, é possível também proporcionar melhores condições de enfrentamento da doença, tanto por paciente como também pela família (VALLE ER, et al., 2001).

A música é uma forma de expressão tanto individual como também coletiva que está presente no cotidiano das pessoas, exercendo um papel de grande relevância dentro dos grupos sociais. A música pode ser conceituada de a arte de combinar os sons de modo a agradar ao ouvido, estimular a inteligência, falar aos sentimentos e comover a alma (BARCELLOS LRM, 2004).

A utilização da música com finalidade terapêutica tem sido uma estratégia para que a criança se sinta melhor em todos os sentidos no ambiente hospitalar, pois ajuda a diminuir a dor e a ansiedade, tão comuns no processo de hospitalização. Halstead MT e Roscoe ST (2007) mostraram que as técnicas utilizada para execução da música auxiliam o humor, aumentam a expressão de sentimentos, dentre muito outros efeitos benéficos, também destaca a sua influência para a qualidade de vida.

Esse tipo de terapia pode ser trabalhada com pacientes em diferentes as faixas etárias, para promover sua saúde e bem-estar, e ainda, favorecer o trabalho interdisciplinar, complementando o tratamento específico de cada patologia, respeitando os princípios éticos. Assim, as crianças na UTI têm mais chances de evoluírem satisfatoriamente no processo de recuperação de seu estado patológico. Visto que a música é considerada pelos estudiosos como uma ferramenta importante e diferenciada no tratamento dos pacientes, considerando o brincar, a sensibilidade e o lúdico (SILVA GH e PIOVESAN JC, 2020). 
Amparado pelo fato de se saber dos benefícios causados pela música no organismo humano, o presente estudo tem como objetivo verificar os efeitos da musicoterapia associada ao seguimento terapêutico às crianças que estão internadas na Unidade de Terapia Intensiva (UTI) pediátrica do Hospital Universitário em um município do Sul Minas Gerais.

\section{MÉTODOS}

Trata-se de um estudo quase experimental, com abordagem quantitativa e analítica. O estudo foi realizado na Unidade de Terapia Intensiva Pediátrica e em enfermaria de pediatria de um Hospital Universitário, localizado no Sul de Minas Gerais. O estudo propõe a aplicação de uma prática não farmacológica que possa ser auxiliar na redução da dor e do estresse, bem como favorecer as respostas imunológicas, as respostas hemodinâmicas através da musicoterapia.

Participaram do estudo crianças com idade gestacional acima de 38 semanas, hospitalizadas, na faixa etária de 0 à 36 meses, sem déficit auditivo, em oxigenoterapia (FiO2 $\leq 30 \%$ ), em ventilação espontânea ou mecânica assistida, submetidas a procedimentos invasivos nas últimas 6 horas e que os pais tenham assinado o Termo de Consentimento Livre e Esclarecido (TCLE), conforme resolução n.466/2012 foi explicado aos pais os objetivos da pesquisa, garantido a eles risco mínimo aos seus filhos e o sigilo de informações referentes as suas identidades (BRASIL, 2012) e adotado por critério de exclusão crianças com déficit auditivo detectado no teste da orelhinha ou não responsivo ao reflexo cócleo-palpebral, idade gestacional inferior a 38 semanas, $\mathrm{FiO} 2>30 \%$, sedação e analgésico contínua e ventilação mecânica em modo controlado.

Foram realizadas sessões de 40 minutos, no período de cinco dias, intervenção de musicoterapia. As músicas selecionadas foram do tipo clássica, repertório "Mozart baby" e observado dados hemodinâmicos durante a exposição a música. Os dados monitorados foram a frequência cardíaca ( $F C)$, frequência respiratória (FR), saturação de oxigênio, temperatura axilar e escala de dor.

Mediante a intervenção, as crianças foram submetidas ao exame de Eletromiografia (RMG), no 1일 e no $5^{\circ}$ dia da intervenção, considerados os resultados no $1^{\circ}$ minuto e com $30^{\circ}$ minutos de sessão, eletrodos dispostos em Bíceps no membro superior esquerdo (MSE), nos respectivos dias foi coletado a amilase salivar para análise, para leitura utilizou-se o aparelho Coroco Meter (Nipro Corporation - Japão).

Vale ressaltar que as caixas de sons utilizadas para a intervenção estavam em concordância com as Normas Técnicas nำ10.152 (ASSOCIAÇÃO BRASILEIRA DE NORMAS TÉCNICAS, 1990) para unidade de terapia intensiva com o volume do som entre 35 a $45 \mathrm{~dB}$.

Para os dados hemodinâmicos foi utilizado um formulário estruturado previamente elaborado pelo pesquisador, contendo as variáveis: sexo, idade, diagnóstico, procedimentos invasivos nas últimas seis horas, FC, FR, saturação de oxigênio e escala de dor. O estudo foi submetido ao Comitê de Ética em Pesquisa da Universidade José do Rosário Vellano, protocolo experimental aprovado sob o parecer no 3.084.727.

\section{RESULTADOS}

Após avaliação do estudo verificou que os valores hemodinâmicos sofreram influência positiva sob sessão de música. Vale ressaltar que as sessões foram conduzidas por músicas do tipo ondas alfas, para esse tipo de ondas as utilizadas foram clássicas instrumentais e o repertório "Mozart Baby". Observa-se na tabela que os dados referentes a temperatura axilar, FR, FC e dor, com evidências de significância pvalor $<0,05$. Pelos achados verificou que a $F R$ foi influenciada em todas as crianças com redução dos parâmetros de 45,6, 54,6, 57,2, 41,2 a média para 38,4, 50, 43,6, 33,6 incursões respiratórias por minuto respectivamente. Estatisticamente apresenta nível de significância de $p$-valor 0,01 . Ainda considerando 0 sistema nervoso central (SNC) centro respiratório e cardiovascular a variável FC apresentou nível de significância p-valor< 0,01, com médias de 150,4, 146, 156,4, 137,6 para 131,2, 127,4, 137,2, 117,2 
batimentos por minuto. Em relação a temperatura corporal, essa influenciada por fatores intrínsecos e extrínsecos não sofreu alteração em seus parâmetros ainda que com redução numérica de suas médias (Tabela 1).

Os parâmetros de saturação de oxigênio, considerando a monitorização por aparelho contínuo, apresentou-se melhora em uma criança e nas demais crianças apresentaram dentro dos parâmetros de normalidade, ou seja, de 92 - 98\%. Com o propósito de aliviar a dor sob terapia não farmacológica, a música evidenciou nível de significância $p$-valor menos que 0,01 . A considerar a criança N3 de 4 meses de vida a dor foi reduzida de forma drástica com p-valor 0,00003 (Tabela 1).

Tabela 1 - Dados hemodinâmicos em 5 dias de intervenção de musicoterapia de 4 crianças submetidas a intervenção da música nas unidades de terapia intensiva e enfermaria de pediatria.

\begin{tabular}{|c|c|c|c|}
\hline \multirow{2}{*}{ Grupo } & \multicolumn{2}{|c|}{ Média } & \multirow{2}{*}{ p-Valor } \\
\hline & Início & Término & \\
\hline Tax.N1 & 36,7 & 36,36 & 0,0625 \\
\hline SatO-N1 & 97,6 & 97,6 & 1 \\
\hline FR-N1 & 45,6 & 38,4 & $0,015448^{*}$ \\
\hline FC- N1 & 150,4 & 131,2 & $0,006311^{* *}$ \\
\hline DOR- N1 & 2,6 & 0,2 & $0,019375^{\star}$ \\
\hline Tax.N2 & 36,8 & 36,42 & 0,131182 \\
\hline SatO.N2 & 94,2 & 96,8 & 0,182745 \\
\hline FR.N2 & 54,6 & 50 & 0,330565 \\
\hline FC.N2 & 146 & 127,4 & $0,006622^{* *}$ \\
\hline DOR.N2 & 3,8 & 1,2 & $0,001174^{\star *}$ \\
\hline Tax.N3 & 36,9 & 36,52 & 0,051941 \\
\hline SatO.N3 & 89,2 & 95,4 & $0,001199^{* *}$ \\
\hline FR.N3 & 57,2 & 43,6 & $0,027351^{*}$ \\
\hline FC.N3 & 156,4 & 137,2 & $0,012781^{*}$ \\
\hline DOR.N3 & 4,4 & 1 & $0,00003^{\star * *}$ \\
\hline Tax.N4 & 36,62 & 36,52 & 0,672016 \\
\hline SatO.N4 & 96,6 & 97,8 & 0,290171 \\
\hline FR.N4 & 41,2 & 33,6 & $0,019825^{\star}$ \\
\hline FC.N4 & 137,6 & 117,2 & $0,01349^{*}$ \\
\hline DOR.N4 & 2,6 & 0,2 & $0,019375^{\star}$ \\
\hline
\end{tabular}

Legenda: $\mathbf{N}$ - crianças participantes. Teste-t: duas amostras presumindo variâncias diferentes: ${ }^{*} \mathrm{P}<0,05$ - Significativo, ${ }^{* *} \mathrm{P}<0,01$ - muito significativo, ${ }^{* * *} \mathrm{P}<0,001$ Extremamente significativo.

Fonte: Silva ACP, et al., 2019. 
Em relação a eletromiografia, a música de forma indireta provocou uma alteração nos disparos de impulso elétrico e por resultado verificou a influência na taxa do disparo para as fibras musculares, como pode ser observado nas médias de início e término, com nível de significância p-valor menor 0,05 (Tabela 2).

Tabela 2 - Média de disparos elétricos registrados em 6 momentos nas quatro crianças sob intervenção da musicoterapia em unidade de terapia intensiva e enfermaria de pediatria.

\begin{tabular}{lccl}
\hline \multirow{2}{*}{ Grupos } & \multicolumn{2}{c}{ Média } & \multirow{2}{*}{ p-Valor } \\
\cline { 2 - 3 } N1 & Início & Término & \\
N2 & 19,56 & 14,80 & 0,122457 \\
N3 & 17,94 & 23,66 & $0,013925^{*}$ \\
N4 & 14,29 & 14,74 & 0,561248 \\
\hline
\end{tabular}

Legenda: $\mathbf{N}$ - crianças participantes. Teste-t: duas amostras presumindo variâncias diferentes: ${ }^{*} \mathrm{P}<0,05$ - significativo.

Fonte: Silva ACP, et al., 2019.

Como parte da proposta terapêutica, foi avaliado o nível de estresse que a criança estava exposta, por meio da dosagem da amilase salivar, importante biomarcador. Ressalta aqui que essa atividade enzimática em crianças menores de dois anos pode não ocorrer (Tabela 3).

Tabela 3 - Amilase salivar em quatro crianças submetidas a intervenção de música clássica.

\begin{tabular}{lcccc}
\hline \multicolumn{5}{c}{ Amilase salivar } \\
\hline N1 & $\begin{array}{c}\text { 10 Dia } \\
\text { (Início) }\end{array}$ & $\begin{array}{c}\text { 10 Dia } \\
\text { (Término) }\end{array}$ & $\begin{array}{c}\text { 50 Dia } \\
\text { (Início) }\end{array}$ & $\begin{array}{c}\text { 50 Dia } \\
\text { (Término) }\end{array}$ \\
N2 & 5 & 6 & 5 & 3 \\
N3 & 4 & 4 & 5 & 3 \\
N4 & 7 & 6 & 7 & 4 \\
\hline
\end{tabular}

Legenda: $\mathbf{N}$ - crianças participantes.

Fonte: Silva ACP, et al., 2019.

Verificou que a atividade enzimática esteve mais evidente no primeiro dia de exposição a música e com os dias sequenciais de ambientalização ao tipo de musicalidade, verificou redução em todas as crianças, porém sem nível de significância estatística, p-valor maior 0,05.

\section{DISCUSSÃO}

Mediante os dados do estudo foi possível verificar que a música consegue modificar parâmetros fisiológicas, como da FC, FR, alívio da dor e relaxamento muscular. Assim, estudo desenvolvido por Franco M e Rodrigues AB (2009) destaca que a música tem exercido um papel universal para o desenvolvimento e funcionamento do organismo humano. É válido mostrar que o homem é um conjunto de reações físicas e químicas, com repercussões emocional e comportamental e a música pode ser um auxílio para melhor direcionar em totalidade positiva tais respostas no indivíduo. 
A frequência cardíaca pode ter sido influenciada pela música via SNC, resultados semelhantes foram descritos por Puggina e Silva. Em seu estudo os autores destacam que a partir de impulsos transmitidos pelo sistema sensorial- audição, via vestíbulo coclear levando a atividade parassimpática com inibição da resposta simpática e por resultado, a redução da frequência cardíaca (PUGGINA ACG e SILVA MJP, 2009).

No presente estudo verificou a redução da frequência respiratória durante a musicalização e resultado semelhante foi observado no estudo desenvolvido por Hatem TP, et al. (2006) que comparou grupos de crianças cardiopatas pós cirúrgicos.

Estudo desenvolvido em 2011 mostrou que a música influenciou a frequência cardíaca e respiratória dentro dos parâmetros de normalidade em pacientes com câncer. Os participantes dessa pesquisa também evidenciaram melhorias da ansiedade, dor, humor (BRADT J, et al., 2011).

Com a expansão da música pode-se verificar que essa vem ocupando lugar dentro da área da saúde, enquanto um processo interpessoal, que utiliza a música e todas as suas facetas para ajudar 0 cliente/paciente a melhorar, recuperar ou manter a saúde (BRITO TA, 2001).

Bruscia K (2000) afirma que a utilização da música para fins terapêuticos deve seguir um processo sistematizado, onde se utiliza a música e seus elementos para atingir objetivos terapêuticos como a prevenção, manutenção e recuperação da saúde física e mental. Com objetivos bem definidos, para a aplicação da música considera importante a experiência musical do ser humano, pois afeta muitas de suas facetas.

Outrossim, deve se levar em consideração o conceito de saúde ampliado estabelecido pela Organização Mundial de Saúde. O conceito de saúde, além de mostrar a multidimensionalidade, abrange não somente uma atuação curativa, mas também uma atuação preventiva e promotora. A atuação, que tem em vista a promoção da saúde, é um campo incipiente para o musicoterapeuta, pois ainda existem fatores que dificultam o acesso a seus conhecimentos e serviços (MOLINA RCM, et al., 2014).

Estudos descrevem a influência da música e um de seus conceitos sob uma visão médica, enxergando o ser humano como um organismo, a doença ou incapacidade como um resultado de entidades separadas e que estão localizadas no corpo do paciente. Por outro lado, a música se encontrava sob uma visão idealística acerca do seu "poder curativo" (RUUD E, 1990).

$\mathrm{Na}$ atualidade são encontradas boas perceptivas e meios para que a atuação promotora da saúde se concretize. Conta-se hoje com uma mudança de paradigma da ciência, que traz um novo olhar holístico e humanista relacionado ao "ser", com a ampliação da consciência sobre a necessidade da busca por melhor qualidade de vida, e com o fácil acesso à informação. Com relação aos meios, há um constante desenvolvimento teórico e técnico da musicoterapia baseado na concepção humanista que viabiliza tal atuação.

A música e seus efeitos terapêuticos podem influenciar a saúde e o comportamento humano. Por séculos a utilização terapêutica da música vem sendo registrada em diversos documentos históricos de culturas distintas (HATEM TP, et al., 2006). Utilizar a música para alívio da dor não é algo novo, estudo desenvolvido por Curtis $S$, et al. demonstrou que participantes no seu estudo evidenciaram alívio da dor, conforto físico, relaxamento e contentamento após ouvir músicas (CURTIS S, 1986).

A música pode ser um recurso para o cuidado de enfermagem, pois sua utilização apresenta uma diversidade de possibilidades de usos, como estimular a interação e participação. Dentre suas vantagens destaca a redução da ansiedade, da dor, da irritabilidade, do medo, da angústia, aumento da autoestima e da memória, integração social, dentre outros (SILVA KG, et al., 2017).

Sabe-se que o uso da música como ferramenta complementar à promoção da saúde tem sido recentemente utilizado em ambiente hospitalar. A música tem sido utilizada no tratamento de doenças somáticas e mentais. A utilização dessa estratégia está fomentado em práticas com evidências científicas para o manejo da dor, da ansiedade e do estresse emocional, entre outras condições (VALLE ER, et al. 2001; BRITO TA, 2001). Pesquisas comprovaram que a música quando utilizada como estratégia para 0 
cuidado pode agir diretamente no corpo humano, sobre o desconforto, aliviando níveis de ansiedade, diminuindo e auxiliando na tolerância da dor (RUUD E, 1990). O recém-nascido prematuro, durante a hospitalização, fica exposto à prática de técnicas e procedimentos invasivos e potencialmente dolorosos que poderão impactar sua qualidade de vida e desenvolvimento neuropsicomotor (MOLINA RCM, et al., 2014).

Foi observado a influência da música, capaz de promover o relaxamento levando a criança a sonolência, atingindo o estágio REM do sono durante a intervenção. Muitas deles chegavam a despertar após o término da intervenção. Tais dados corroboram com Evans D (2002) que descreve em seu estudo que a música para fins analgésicos tem alcançado destaque, pois se mostra eficiente em diminuir a ansiedade e aumentar o grau de relaxamento de pacientes graves em qualquer faixa etária. $O$ autor esclarece que esse resultado obtido a partir da música é benéfico até mesmo aos prematuros

Acredita-se que a sonoridade ativa diversos mecanismos cerebrais, com isso ela pode provocar diferentes respostas associadas ao ritmo, letra, experiências anteriores e tais ativações significa transmissão de impulsos elétricos por células neuronais, assim diferentes partes do corpo serão estimulados e pelo que se mostrou nesse estudo fibras musculoesqueléticas foram estimuladas levando ao relaxamento muscular. Estudo corrobora com esses achados e afirmam que a música é vista pelos pacientes como uma atividade motivadora, visto que essa estratégia contribui para o aumento da autoestima e descontração do ambiente em que esses pacientes se encontram. Assim pode ser aplicada para minimizar os agravos provenientes da situação emocional advindos do processo de hospitalização e terapia (MOURA CC, et al., 2012).

Além disso, pode contribuir para uma melhor comunicação do profissional com a criança. A música permite um tipo de comunicação também para a criança quando essa tem em seu universo o brincar como atividade fundamental para seu desenvolvimento. É importante compreender que a música sempre esteve inserida no cotidiano humano e de formas diferentes estando presente em todas as culturas, utilizada como recurso para diferentes fins. Pela ótica de uma revisão de literatura realizada em 2006, os autores consideraram a intervenção musical benéfica, pois desencadeia mudanças fisiológicas e psicológicas independente da faixa etária do paciente, constitui assim um recurso eficaz para qualificar o cuidado à criança hospitalizada, ressaltado ainda que trata de uma prática de baixo custo, não-farmacológica e nãoinvasiva, com possibilidades de promoção dos processos de desenvolvimento e a saúde da criança, da família e dos trabalhadores (FERREIRA CCM, et al., 2006).

No decorrer de vários séculos, a música vem sendo aplicada de forma intuitiva para intervir no tratamento terapêutico, no resgate ao equilíbrio, tanto do corpo como do espírito, especialmente quando outras ações não se mostravam eficientes. Ela, aparentemente, vem imitar as mais diversas características presentes na linguagem, transmitindo assim, as mais diversas emoções. Sendo elas semelhantes a comunicação verbal, mas de uma forma não referencial e não específica. Esta também vem mobilizar as mais diversas regiões neurais além da área da linguagem, a música é captada pelas estruturas "cerebrais primitivas" envolvidas com a motivação e a emoção. Assim, a literatura mostra como a música pode ser um complemento terapêutico, pois os efeitos da música são desencadeados inicialmente pela via tálamos. É importante ressaltar que a membrana auditiva possui inúmeras células sensoriais responsáveis pela condução do impulso sonoro ao córtex cerebral. Nesse local, o som atua no complexo amigdaloide, relacionado a situação de estresse, medo ou tristeza. Tal impulso é conduzido para o hipotálamo e pelo eixo hipotámo-hipofisário estimula-se a liberação da substância Adrenocorticotrópico (ACTH), importante estimulante das suprarrenais para a síntese e liberação do cortisol (GUYTON AC e HALL JE, 2017).

Sabe-se que o cortisol pode ser dosado no sangue, saliva e urina, e que se elevado pode sinalizar processos de estresse oxidativo no organismo. O paciente, em processo de hospitalização evidencia certa grau de ansiedade, essa ansiedade, assim como o estresse, provoca respostas biológicas, envolvendo diversos biomarcadores, dentre eles, cortisol, facilmente identificado na saliva (RODRIGUEZ DL, et al., 2011). Dentre os vários biomarcadores para se avaliar a o estresse, a alfa-amilase salivar é muito utilizada. Vale ressaltar que a alfa amilase em crianças de até dois anos tem sua atividade enzimática praticamente zerada. Se dosada na urina, considera para a determinação do clearence o diagnóstico diferencial das 
causas de hiperamilasemia e os valores expresso em porcentagem de 1,4 a 3,8\% (SECRETARIA DE SAÚDE DO ESTADO DE MINAS GERAIS, 2009).

A música tem estado presente, em vários rituais importantes na vida humana perante a sociedade. Havendo melodias apropriadas para casamento, aniversário, músicas de ninar, músicas para festas cívicas, para rituais religiosos e para funerais. Assim, a música é encontrada em todas as culturas do mundo, nas mais primitivas e desprovidas de conhecimentos tecnológicos. Estudo tem destacado suas várias influências sobre o corpo humano dentre elas a liberação de endorfinas, reduzindo episódios de dor, assim, a música pode ser um importante recurso de comunicação e contribuir para a redução de transtornos psicoemocionais. Seu mecanismo ondulatório para a excitação e relaxamento do córtex cerebral já está bem estabelecida na ciência, com destaque para a via talâmica, com atuação também no processamento do pensamento, cognição e estado emocional (YANG ET, et al., 2012).

Para Bergold LB, et al. (2016), os benefícios da música agregam a manifestação de diferentes afetos dos quais pode destacar a redução do sentimento de solidão, promoção de sentimentos de estar em lugar agradável, bem estar geral e segurança. A música é capaz de promover conforto espiritual e a ideia de cura. Em seu estudo, os autores afirmaram que os pacientes submetidos a intervenção da música relataram sentir paz interior, alegria, tranquilidade e relaxamento físico.

A estabilização dos dados hemodinâmicos, relaxamento muscular e comportamento de calmaria, resultando em sono tem relação com a abordagem feita por Juslin e Västfjall ao descrever os mecanismos psicológicos, visto que ocorre por meio do estímulo primário, a audição. Os autores afirmam que as respostas induzidas pela escuta há a ativação do cérebro, especificamente de certas regiões como o tálamo, hipocampo, amigdala, córtex pré-frontal, córtex orbitofrontal. E a partir dessas regiões ativadas ocorre a determinação das sensações de prazer ou desprazer; excitação ou relaxamento (JUSLIN PN, VÄSTFJALL D, 2008), além disso, em outro estudo desenvolvido mostrou que a música provocou diminuição na tensão, sentimento de tristeza, fadiga e aumento da clareza mental (KEMPER KJ, et al., 2005).

\section{CONCLUSÃO}

A influência da música no contexto da hospitalização é algo que humaniza e enriquece a todos que a usufrui. O hospital é um local de sobrecarga emocional, tanto para os familiares como para os profissionais, a música com prática não farmacológica se caracteriza como uma ferramenta de humanização, pois auxilia em sentimentos positivos e como ferramenta assistencial, pois acarreta alterações fisiológicas, principalmente nos sinais vitais, benéficas para as crianças hospitalizadas. Apesar de utilizada como terapia desde os tempos antigos e trazer inúmeros benefícios para a saúde e bem-estar do indivíduo ainda é pouco utilizada na assistência. Conclui-se que a música tem potencial de estimular diferentes áreas cerebrais e pode ser incluída como ferramenta complementar para auxiliar em segmentos terapêuticos.

\section{REFERÊNCIAS}

1. ASSOCIAÇÃO BRASILEIRA DE NORMAS TÉCNICAS - ABNT, NBR 10152. Níveis de ruído para conforto acústico, 1990.

2. AZEVEDO E; LIMA RAG. Assistência à criança hospitalizada: o trabalho do clown como recurso de comunicação da enfermagem pediátrica. In: 10ํㅗำósio de Iniciação Científica da Universidade de São Paulo; 2002 nov 5; Ribeirão Preto (SP): Universidade de São Paulo, 2002.

3. BARCELLOS LRM. Musicoterapia: alguns escritos. Rio de Janeiro: Enelivros, 2004.

4. BATISTA MA. Presença do Sagrado em um momento crítico: Internação em uma Unidade de Terapia Intensiva. Revista Brasileira de Enfermagem. 54(5):579-585. Brasília - DF. 2004.

5. BERGOLD LB, et al. A utilização da música na humanização do ambiente hospitalar: interfaces da musicoterapia e enfermagem. 2016. Revista Brasileira de Musicoterapia, 11: 56-70.

6. BRADT J, DILEO C, GROCKE D, MAGILL L. Music interventions for improving psychological and physical outcomes in cancer patients. Cochrane database of systematic reviews. 2011; (8):CD006911

7. BRASIL. Conselho Nacional de Saúde. Resolução № 466, De 12 De Dezembro De 2012. Resolve: Aprovar as seguintes diretrizes e normas regulamentadoras de pesquisas envolvendo seres humanos, 2012. 
8. BRITO TA. Koellreutter educador: o humano como objetivo da educação musical. São Paulo. 2001.

9. BRUSCIA K. Definindo Musicoterapia. Rio de Janeiro: Enelivros, 2000. 312p.

10. CASTRO NETO A. As fases turbulentas da hospitalização. Pediatria Moderna, 2000; 36(4): 245-7.

11. CURTIS S. The effect of music on pain relief and relaxation of the terminally ill. Journal Music Therapy. 24(1):1024, 1986.

12. EVANS D. The effectiveness of music as an intervention for hospital patients: a systematic review. Journal of Advanced Nursing, 37:8-18, 2002.

13. FERREIRA CCM, REMEDI PP, LIMA RAG. A música como recurso no cuidado à criança hospitalizada: uma intervenção possível? Revista Brasileira de Enfermagem. 2006; 59(5): 689-693.

14. FRANCO M, RODRIGUES AB. A música no alívio da dor em pacientes oncológicos. Einstein. 7(2): 147-51, 2009.

15. GUYTON AC, HALL JE. Tratado de fisiologia médica. 13 ed. Rio de Janeiro: Elsevier, 2017.

16. HALSTEAD, M. T.; ROSCOE, S. T. Restoring the spirit at the end-of-life: music as an intervention for oncology nurses. Clinical journal of oncology nursing. 6(6): 332-6, 2007

17. HATEM TP, LIRA PIC, MATTOS SS. Efeito terapêutico da música em crianças em pós-operatório de cirurgia cardíaca. Journal Pediatric. Porto Alegre. 82 (3). 2006.

18. JUSLIN PN, VÄSTFJALL D. Emotional responses to music: the need to consider underlying mechanisms. Behavioral and Brain Sciences, 31(5):559-621, 2008.

19. KEMPER KJ, DANHAUER SC. Music as therapy. Southern Medical Journal; 98(3): 282-8, 2005.

20. MOLINA RCM, HIGARASHI IH, MARCON SS. Importância atribuída à rede de suporte social por mães com filhos em unidade intensiva. Escola Anna Nery. Rio de Janeiro. 18(1). 2014.

21. MOURA CC, RESCK ZMR, DÁZIO EMR. Atividades lúdicas realizadas com pacientes portadores de neoplasia internados em Hospital Geral. Revista da Rede de Enfermagem do Nordeste - Rene. 2012; 13(3):667-76.

22. PUGGINA ACG, SILVA MJP. Sinais vitais e expressão facial de pacientes em estado de coma. Revista Brasileira de Enfermagem. Brasília. 62 (3): 435-441. 2009.

23. RODRíGUEZ DL, et al. The application of Reiki in nurses diagnosed with Burnout Syndrome has beneficial effects on concentration of salivary $\operatorname{lgA}$ and blood pressure. Revista Latino-Americana de Enfermagem, 19(5):1132-1138, 2011.

24. RUUD E. Caminhos da musicoterapia. São Paulo. 1990.

25. SECRETARIA DE SAÚDE DO ESTADO DE MINAS GERAIS. Protocolo Clínicos dos exames laboratoriais. Universidade Federal de Minas Gerais. 2009.

26. SILVA GH, PIOVESAN JC. Música e Alegria: uma Prática Humanizada para Crianças Hospitalizadas. Revista Vivências Erechim, jan./jun., 2020. 16(30):127-144.

27. SILVA KG, TAETS GGC, BERGOLD LB. A utilização da música em uma unidade pediátrica: contribuindo para a humanização hospitalar. Revista de enfermagem UERJ, Rio de Janeiro, 2017; 25: e26265.

28. VALLE ER. (Org). Psico-Oncologia Pediátrica. Casa do Psicólogo, São Paulo. 2001.

29. YANG, E. T et al. The effect of music therapy on hospitalized psychiatric patients' anxiety, finger temperature, and electroencephalography: a randomized clinical trial. Biological Research for Nursing, 14(2): 197-206, 2012. 